\title{
Multivariate analysis identify new loci associated with meat productivity and carcass traits in sheeps (Ovis aries)
}

\author{
Alexander S. Zlobin \\ Kurchatov Genomic Center of ICG \\ SB RAS, Novosibirsk, Russia \\ zlobin@bionet.nsc.ru \\ Tatiana I. Aksenovich \\ L.K. Ernst Federal Science Center \\ for Animal Husbandry, \\ Dubrovitsy, Moscow Region, Russia
}

\author{
Natalia A. Volkova \\ L.K. Ernst Federal Science Center \\ for Animal Husbandry, \\ Dubrovitsy, Moscow Region, Russia \\ Yakov A. Tsepilov \\ Laboratory of Theoretical and Applied \\ Functional Genomics \\ Novosibirsk State University \\ Novosibirsk, Russia
}

\author{
Pavel M. Borodin \\ L.K. Ernst Federal Science Center \\ for Animal Husbandry, \\ Dubrovitsy, Moscow Region, Russia
}

\begin{abstract}
We performed the search of new loci associated with meat productivity and carcass traits in sheep using novel multivariate approaches. We found 4 new loci associated with traits of interest and added more than $\mathbf{3 0}$ loci to our previously published database.
\end{abstract}

Keywords - sheep, meat productivity traits, carcass traits

Introduction

Sheep breeding is one of the most important branches of agriculture. Currently, the most popular product of sheep breeding is mutton. For this reason, the modern market demands the breeds or breed types of sheep characterized by good viability, high growth rate, good meat qualities, and ability to effectively use feed. The solution of the problem of production of pedigree sheep of competitive quality (both on the domestic and world markets) can only be achieved through the shift of selection to a qualitatively new level from selection by phenotype to the selection at the gene and genomic level. Genome-wide association studies (GWAS) is a modern, agnostic, data-driven method for identifying QTL associated with different traits. Identification and mapping of genes that affect phenotypic variability in economically important quantitative traits, determining the growth, development and reproductive potential of animals. Unfortunately, today we don't have a full picture of QTLs and genes associated with traits of interest. Filling the gap in our knowledge can be done by performing new GWAS on these traits or reprocessing previously obtained results of GWAS (such as published summary statistics) using novel powerful methods. One of the method to do reprocessing of GWAS results is MANOVA (Multivariate ANalysis Of VAriance). This method allows to find new loci associated with traits of interest using summary statistics. This project focused on application existed methods of multivariate analysis for searching for new loci associated with meat productivity and carcass traits in sheep.

\section{Materials and methods}

For this project, we used summary statistics from previously published GWAS for 56 traits associated with meat productivity and carcass traits in sheep.[1] We performed multivariate analysis for 8 new multivariate traits formed from 48 univariate original traits. Each multivariate trait is a combination of several univariate traits grouped based on the similarity effect on the organism.
The results of multivariate analysis have greater power that regular univariate analysis and usually depicts loci with high level of pleiotropicity between this group of traits. We used the adapted MANOVA method for $\mathrm{z}$-statistics from Stephens et al. 2013 [2]. Number of SNPs in analisys was more than 450000. The locus was defined as $+-250000 \mathrm{bp}$ from top SNP. We considered locus significantly associated in univariate analysis if its $\mathrm{p}$-value was $<0.05 /\left(\mathrm{N}_{\mathrm{snp}} * 48\right)$ and in multivariate analysis if its p-value was < $0.05 /\left(\mathrm{N}_{\text {snp }} *(48+8)\right)$. We considered locus as new if it didn't present in our previously published database.

\section{Results}

We found 4 new loci associated with one multivariate trait related to weight: rs402280290, rs418394153, rs420734786 and rs193632759. These top associated SNPs were missense variant (rs193632759), intron variant (rs420734786) and two - interegenic variants (rs402280290 and rs418394153). The nearest genes were LOC105603262 (rs402280290), SETBP1 (rs418394153), DOCK8 (rs420734786) and MASP1 (rs193632759). In addition, we found 39 new loci which were associated with multivariate and/or univariate traits and were not presented in our database contained loci associated with meat and growth traits for sheep. [3] As a result of this work we found new loci associated with traits of interest and add these new loci in our database. This information can be useful for further association studies and preliminary estimation of genetic variability for economically important traits in different breeds.

\section{ACKNOWLEDGMENT}

The work of $\mathrm{AZ}$ was funded by the Kurchatov Genomics Center of IC\&G (075-15-2019-1662). The work of NV, PB, TA and YT was supported by Russian Science Foundation \#18-1600079 .

\section{REFERENCES}

[1] Bolormaa S. et al. Detailed phenotyping identifies genes with pleiotropic effects on body composition // BMC Genomics. BioMed Central, 2016. Vol. 17, № 1. P. 224.

[2] Stephens M. A Unified Framework for Association Analysis with Multiple Related Phenotypes // PLoS One / ed. Emmert-Streib F. 2013. Vol. 8, № 7. P. e65245.

[3] Zlobin A.S. et al. Recent advances in understanding genetic variants associated with growth, carcass and meat productivity traits in sheep ( Ovis aries ): an update // Arch. Anim. Breed. 2019. Vol. 62, № 2. P. 579-583. 